\title{
新型红外非线性光学晶体探索取得新进展
}

\author{
周慧敏，吴立明* \\ 北京师范大学化学学院, 北京 100875 \\ * 联系人, E-mail: wlm@bnu.edu.cn
}

\section{The exploration of new infrared nonlinear optical crystals}

\author{
Huimin Zhou \& Liming $\mathrm{Wu}^{*}$ \\ College of Chemistry, Beijing Normal University, Beijing 100875, China \\ * Corresponding author, E-mail: zslin@mail.ipc.ac.cn \\ doi: 10.1360/N972019-00076
}

波长范围在2 20 $\mu \mathrm{m}$ 之间的中远红外激光在军事和民 用方面有着极其重要的应用价值(红外对抗、激光制导、激 光手术、大气监测等). 产生中远红外激光的方法包括化学 激光器、气体激光器、自由电子激光器、量子级联激光器 和全固态激光器等方法; 其中全固态激光器具有技术成 熟、调谐范围宽、光束质量好等优点, 是目前产生中远红 外激光的主流方法. 该方法需要用到非线性变频技术对成 熟的近红外激光光源进行频率转换从而获得红外可调谐 激光输出, 其中频率转换器件是一种光功能晶体(非线性 光学晶体). 由于将其应用在红外波段, 称为红外非线性光 学晶体, 此类晶体是该方法的核心部件之一.

目前, 商用的红外非线性晶体仅仅包含 $\mathrm{AgGaS}_{2}, \mathrm{Ag}$ $\mathrm{GaSe}_{2}$ 和 $\mathrm{ZnGeP}$ 等为数不多的几个黄铜矿结构化合物. 由 于存在激光损伤阈值低、高品质晶体制备困难等缺点而远 不能满足高功率和宽波段输出的应用需求, 因此探究新型 优质红外非线性光学晶体是当前国际光电子领域的一个 热点.

硫属化合物在中、远红外区域有高的透过率和强的非线 性光学效应等优点, 是新型红外非线性光学晶体材料的主要 研究对象. 近几年, 国内外多个课题组积极进行探索, 取得 了卓有成效的结果, 发现了包括 $\mathrm{BaGa}_{4} \mathrm{~S}_{7}{ }^{[1]}, \mathrm{BaGa}_{4} \mathrm{Se}_{7}{ }^{[2]}$, $\mathrm{BaGa}_{2} \mathrm{GeSe}_{6}{ }^{[3,4]}, \mathrm{La}_{4} \mathrm{InSbS}_{9}{ }^{[5]}, \mathrm{Na}_{2} \mathrm{ZnGe}_{2} \mathrm{~S}_{6}{ }^{[6]}, \mathrm{CsM}_{3} \mathrm{Se}_{6}(\mathrm{M}=$ $\mathrm{Ga} / \mathrm{Sn}, \mathrm{In} / \mathrm{Sn})^{[7]}, \mathrm{BaHgSe}_{2}{ }^{[8]}$ 等一系列优质新型红外非线性 光学晶体材料. 上述新晶体目前还处于实验室开发阶段, 具体实用性如何有待进一步研究. 2019年1月7日, 中国工 程物理研究院化工材料研究所尹文龙课题组和加拿大阿 尔伯塔大学Arthur Mar课题组 ${ }^{[9]}$ 在Crystal Growth \& Design 上在线发表题为“ $\mathrm{SrCdGeS}_{4}$ and $\mathrm{SrCdGeSe}_{4}$ : Promising infrared nonlinear optical materials with congruent-melting be- haviour”的研究论文, 通过结构设计和元素调控, 在 $\mathrm{Sr}-\mathrm{Cd}-\mathrm{Ge}-\mathrm{Ch}(\mathrm{Ch}=\mathrm{S}, \mathrm{Se})$ 四元体系中首次合成了两种具有优异 红外非线性光学性能的硫属化合物 $\mathrm{SrCdGeS}_{4}$ 和 $\mathrm{SrCdGeSe}_{4}$, 引起广泛关注.

尹文龙课题组和Arthur Mar课题组 ${ }^{[9]}$ 采用高温固相法 合成出 $\mathrm{SrCdGeS}_{4}$ 和 $\mathrm{SrCdGeSe}_{4}$ 小晶体和粉末纯相并精修了 其晶体结构. 这两个同构化合物具有二维层状结构, 属于 正交晶系Ama2空间群(图 1(a)); 紫外可见漫反射光谱表明 其带隙大, 预示着较高的激光损伤阈值. $\mathrm{SrCdGeS}_{4}$ 的带隙 为 $3.36 \mathrm{eV}$, 大于经典材料 $\mathrm{AgGaS}_{2}(2.7 \mathrm{eV})$ 和 新材料 $\mathrm{BaGa}_{4} \mathrm{Se}_{7}(2.6 \mathrm{eV})$, 而 $\mathrm{SrCdGeSe}_{4}$ 带隙与传统经典红外非线 性光学晶体 $\mathrm{AgGaSe}_{2}\left(1.8 \mathrm{eV}\right.$ ) 和 $\mathrm{ZnGeP}_{2}(2.0 \mathrm{eV}$ ) 相当 (图 1(b)); $2090 \mathrm{~nm}$ 处的倍频信号是 $\mathrm{AgGaS}_{2}$ 晶体的 1.7 倍 $\left(\mathrm{SrCdGeS}_{4}\right)$ 和 5.3倍 $\left(\mathrm{SrCdGeSe}_{4}\right)$ (图 1(c), (d)), 并且都可以 实现一类相位匹配. 另外, 难能可贵的是它们都具有同成 分熔融的性质, 且熔点较低 $\left(\mathrm{SrCdGeS}_{4}\right.$ 为 $958^{\circ} \mathrm{C}, \mathrm{SrCdGeSe}_{4}$ 为 $\left.851^{\circ} \mathrm{C}\right)($ 图1(e), (f)), 可以采用布里奇曼法进行高品质晶 体制备. 初步研究结果表明, $\mathrm{SrCdGeS}_{4}$ 和 $\mathrm{SrCdGeSe}_{4}$ 具有优 秀的红外非线性光学性质, 在中、远红外激光频率变换领 域具有潜在的应用价值.

尹文龙课题组和Arthur Mar课题组 ${ }^{[9]}$ 这一工作的意义 在于首次对 $\mathrm{Sr}-\mathrm{Cd}-\mathrm{Ge}-\mathrm{Ch}(\mathrm{Ch}=\mathrm{S}, \mathrm{Se})$ 四元体系开展了以寻找 新型非线性光学晶体材料为目的的探索研究, 并且发现了 $\mathrm{SrCdGeS}_{4}$ 和 $\mathrm{SrCdGeSe}_{4}$ 两种非黄铜矿结构新晶体, 进一步 丰富了红外非线性光学晶体材料数据库. 当然, 我们也不 应忽视, 目前这两个化合物的大尺寸高品质单晶制备还未 成功, 其中远红外谐波输出能力还有待实验证实, 距离实 际应用还有差距. 因此, 对这两个材料的单晶制备和详细 的光学、热学等性质需要作进一步的研究. 

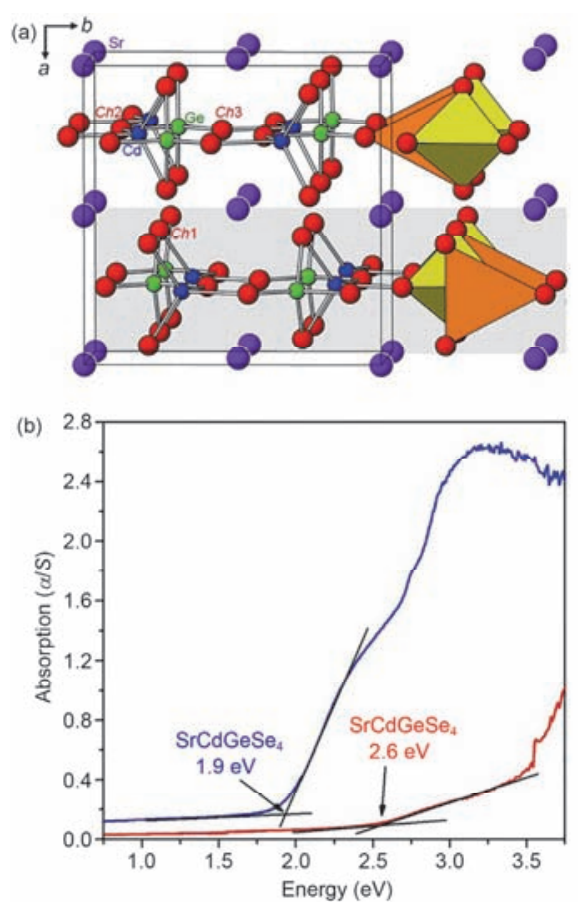
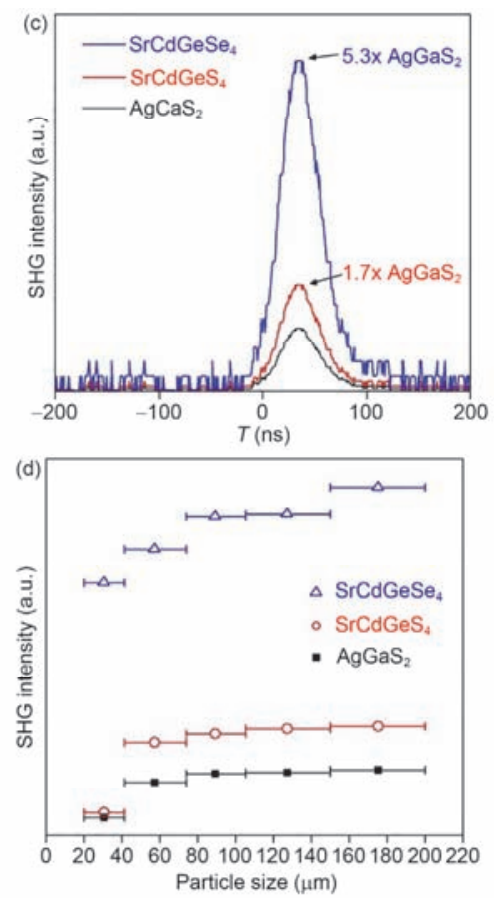
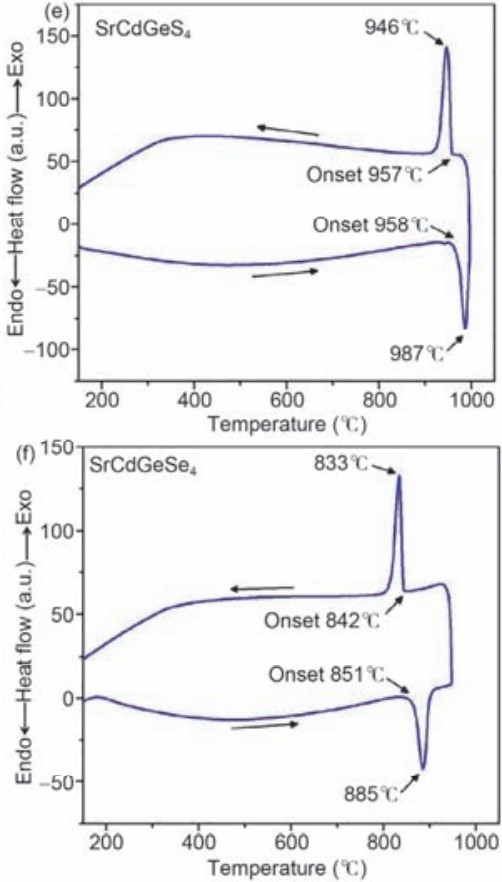

图 1 (网络版彩色) $\mathrm{SrCdGeS}_{4}$, 和 $\mathrm{SrCdGeSe}_{4}$ 的性质表征. (a) $\mathrm{SrCdGeS}_{4}$ 晶体结构图; (b) 紫外可见漫反射光谱; (c) 150 200 $\mu \mathrm{m}$ 粒径范围内 $\mathrm{SrCdGeS}_{4}, \mathrm{SrCdGeSe}_{4}$ 与 $\mathrm{AgGaS}_{2}$ 倍频信号强度对比图; (d) $2090 \mathrm{~nm}$ 的倍频信号与一类相位匹配图; (e) $\mathrm{SrCdGeS}_{4}$ 差热曲线; (f) $\mathrm{SrCdGeSe}_{4}$ 差热曲线 Figure 1 (Color online) Characterization of $\mathrm{SrCdGeS}_{4}$ and $\mathrm{SrCdGeSe}_{4}$. (a) The crystal structure of $\mathrm{SrCdGeS}_{4}$; (b) UV-Vis diffused reflectance spectrum; (c) second harmonic generation intensity of $\mathrm{SrCdGeS}_{4}, \mathrm{SrCdGeSe}_{4}$ and reference $\mathrm{AgGaS}_{2}$ at 150-200 $\mu \mathrm{m}$ particle sizes; (d) phase matching curve under the laser radiation at $2090 \mathrm{~nm}$; (e) thermogravimetry curve of $\mathrm{SrCdGeS}_{4}$; (f) thermogravimetry curve of $\mathrm{SrCdGeSe}_{4}$

\section{参考文献}

1 Lin X, Zhang G, Ye N. Growth and characterization of $\mathrm{BaGa}_{4} \mathrm{~S}_{7}$ : A new crystal for Mid-IR nonlinear. Optics Cryst Growth Des, 2009, 9: 1186-1189

2 Yao J, Mei D, Bai L, et al. $\mathrm{BaGa}_{4} \mathrm{Se}_{7}$ : A new congruent-melting IR nonlinear optical material. Inorg Chem, 2010, 49: 9212-9216

3 Yin W, Feng K, Mei D, et al. $\mathrm{BaGa}_{2} \mathrm{MQ}_{6}(\mathrm{M}=\mathrm{Si}, \mathrm{Ge} ; \mathrm{Q}=\mathrm{S}$, Se): A new series of promising IR nonlinear optical materials. Dalton Trans, 2012, 41: 5653-5661

4 Lin X, Guo Y, Ye N. BaGa $\mathrm{GeX}_{6}(\mathrm{X}=\mathrm{S}$, Se): New mid-IR nonlinear optical crystals with large band gaps. J Solid State Chem, 2012, 195: 172-177.

5 Zhao H, Zhang Y, Chen L. Strong kleinman-forbidden second harmonic generation in chiral sulfide: $\mathrm{La}_{4} \mathrm{InSbS}$. J Am Chem Soc, 2012, 134: 1993-1995

$6 \mathrm{Li} \mathrm{G}, \mathrm{Wu} \mathrm{K}$, Liu Q, et al. $\mathrm{Na}_{2} \mathrm{ZnGe}_{2} \mathrm{~S}_{6}$ : A new infrared nonlinear optical material with good balance between large second-harmonic generation response and high laser damage threshold. J Am Chem Soc, 2016, 138: 7422-7428

7 Lin H, Chen L, Yu J S, et al. Infrared SHG materials $\mathrm{CsM}_{3} \mathrm{Se}_{6}(\mathrm{M}=\mathrm{Ga} / \mathrm{Sn}, \mathrm{In} / \mathrm{Sn})$ : Phase matchability controlled by dipole moment of the asymmetric building unit. Chem Mater, 2017, 29: 499-503

8 Li C, Yin W, Gong P, et al. Trigonal planar $\left[\mathrm{HgSe}_{3}\right]^{4-}$ Unit: A new kind of basic functional group in IR nonlinear optical materials with large susceptibility and physicochemical stability. J Am Chem Soc, 2016, 138: 6135-6138

9 Dou Y, Chen Y, Li Z, et al. $\mathrm{SrCdGeS}_{4}$ and $\mathrm{SrCdGeSe}_{4}$ : Promising infrared nonlinear optical materials with congruent-melting behaviour. Cryst Growth Des, 2019, doi: 10.1021/acs.cgd.8b01649 